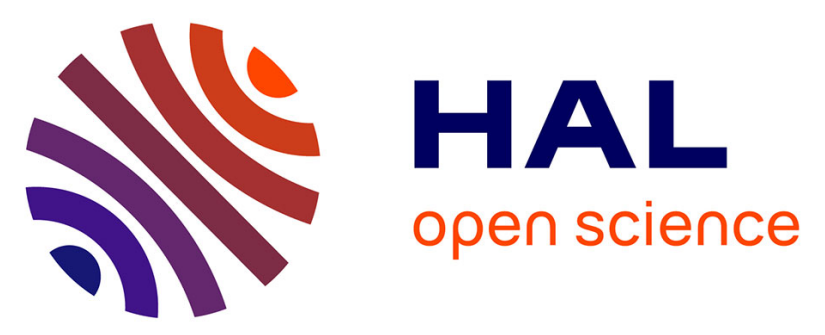

\title{
Caractérisation de la propreté inclusionnaire d'aciers de fonderie par analyse d'images en microscopie électronique à balayage
}

Cécile Nicoli, Jean-François Carton, Alexis Vaucheret, Philippe Jacquet

\section{To cite this version:}

Cécile Nicoli, Jean-François Carton, Alexis Vaucheret, Philippe Jacquet. Caractérisation de la propreté inclusionnaire d'aciers de fonderie par analyse d'images en microscopie électronique à balayage. Metallurgical Research \& Technology, 2019, 116 (5), pp.511. 10.1051/metal/2018133 . hal-02545377

\section{HAL Id: hal-02545377 \\ https://hal.science/hal-02545377}

Submitted on 17 Apr 2020

HAL is a multi-disciplinary open access archive for the deposit and dissemination of scientific research documents, whether they are published or not. The documents may come from teaching and research institutions in France or abroad, or from public or private research centers.
L'archive ouverte pluridisciplinaire HAL, est destinée au dépôt et à la diffusion de documents scientifiques de niveau recherche, publiés ou non, émanant des établissements d'enseignement et de recherche français ou étrangers, des laboratoires publics ou privés. 


\title{
Caractérisation de la propreté inclusionnaire d'aciers de fonderie par analyse d'images en Microscopie Electronique à Balayage
}

\author{
Cécile Nicoli $^{\mathrm{a}, \mathrm{b}, \mathrm{c} *}$, Jean-François Carton $^{\mathrm{b}}$, Alexis Vaucheret ${ }^{\mathrm{a}, \mathrm{c}}$, Philippe Jacquet ${ }^{\mathrm{a}, \mathrm{c}}$ \\ a LaBoMaP, Arts et Métiers ParisTech, 71250 Cluny, France \\ ${ }^{\mathrm{b}}$ CastMetal, 1 Boulevard de la Boissonnette, 42110 Feurs, France \\ c Université de Lyon, ECAM Lyon, INSA-Lyon, LabECAM, F-69005 Lyon, France
}

Mots clés : inclusions, inoculation, comptage inclusionnaire, Microscopie électronique à balayage, aciers faiblement alliés

\section{Résumé}

L'étude porte sur les aciers bas carbone $(0.2 \%)$ faiblement alliés bruts de fonderie. Les différentes opérations effectuées dans le bain liquide de l'acier influent sur la population inclusionnaire; ces inclusions sont elles-mêmes responsables d'une partie des propriétés mécaniques de l'acier. Leur contrôle et leur étude revêtent donc une grande importance.

L'objet de ce travail est de mettre au point une méthode de comptage, d'en évaluer la fiabilité et la reproductibilité ainsi que d'en montrer la limite. D'ordinaire, cette opération est réalisée par microscopie optique, méthode longue à mettre en œuvre et ne couvrant qu'une surface d'échantillon très limitée.

La méthode présentée ici requiert un Microscope Electronique à Balayage (MEB) doté d'une forte stabilité du faisceau d'où l'utilisation d'un canon à émission de champs (FEG). Cette méthode est plus rapide et couvre une surface plus large. Elle est déjà utilisée en sidérurgie pour des aciers laminés mais est beaucoup moins courante pour des contrôles métallurgiques sur des aciers moulés. Pour transposer cette méthode à ce type d'acier il est donc nécessaire de confirmer sa fiabilité et sa répétabilité.

Dans un premier temps, un opérateur définit des critères morphologiques et chimiques sur les inclusions rencontrées dans une zone représentative très réduite. Dans un second temps, un logiciel scanne automatiquement une zone beaucoup plus large et classe les inclusions détectées selon les critères pré-définis (taille, forme, nature).

Afin de statuer sur la répétabilité et la reproductibilité de cette méthode, une étude statistique est réalisée sur une surface test ; cette zone est analysée plusieurs fois dans le but de définir des intervalles de tolérances.

Si les résultats obtenus en terme de population inclusionnaire sont significatifs, il sera alors possible de statuer sur la pertinence des différentes opérations effectuées dans les bains d'acier liquide.

\section{Introduction}

La propreté inclusionnaire est un des enjeux de la métallurgie. Son contrôle peut se faire suite à la fusion du métal lors de la désoxydation du bain liquide, une étape primordiale dans l'élaboration des aciers. Cette étape est accomplie en ajoutant des éléments qui réagissent avec l'oxygène pour former des oxydes [1]. Plusieurs alliages peuvent être utilisés pour la désoxydation [2], tels que le zirconium, le calcium, le manganèse, le silicium ou l'aluminium. Dans cette étude, seul l'aluminium est utilisé comme désoxydant comme c'est le cas dans certaines fonderie d'acier [3]. De plus l'aluminium est un des premiers éléments à réagir avec l'oxygène pour la formation des oxydes, ainsi que nous le montre les diagrammes d'Ellingham [4]. Ainsi les interactions des autres éléments ajoutés seront limitées avec l'oxygène. L'aluminium a également un impact sur le type des inclusions de sulfures [5][6][7]. 
Pour pousser plus loin le contrôle de la propreté inclusionnaire, nous avons fait varier le type et la forme des inclusions en utilisant la méthode d'inoculation. Des éléments chimiques ajoutés en faible quantité (inoculants) dans le bain liquide de l'acier ne modifient pas la nuance mais la répartition inclusionnaire.

Les principaux types d'inclusion détectés sont les oxydes, petits, ronds et noirs, et les sulfures. Il existe trois types d'inclusions de sulfure[8]. Le type I (Figure 1) est sphérique, gris et réparti aléatoirement, et c'est le moins nocif des trois types sur les propriétés mécaniques de l'acier. Le type II (Figure 2) est allongé, gris et aligné plus ou moins de façon discontinue ; c'est le plus nocif pour l'acier. Le type III (Figure 3) est polyédrique, gris et situé aux joints des grains de solidification.

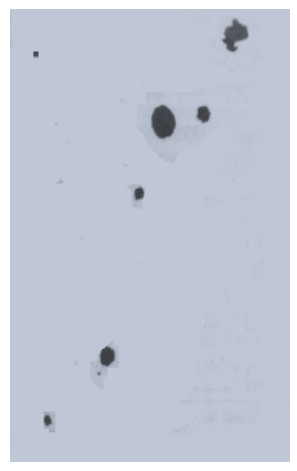

Figure 1 : Exemple

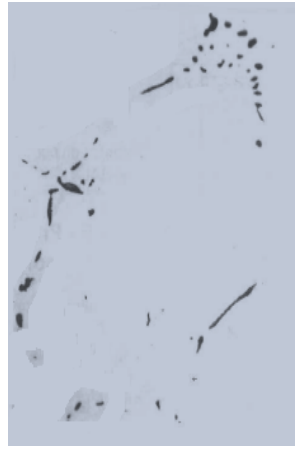

Figure 2 : Exemple

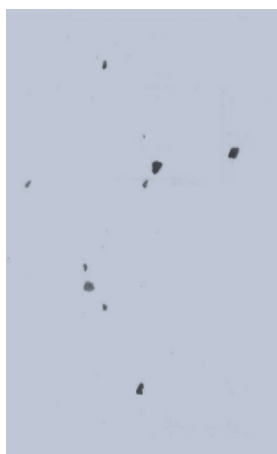

Figure 3 : Exemple

d'inclusion de sulfure type I, d'inclusion de sulfure type II, d'inclusion de sulfure type $\mathbf{x 8 0 0}[9]$ $\mathbf{x 5 0 0}[9]$

L'observation de la composition inclusionnaire de l'acier s'est faite sur un acier faiblement allié traité par différentes teneur d'aluminium et avec différents types d'inoculant. La technique utilisée requiert un microscope électronique à balayage (MEB-FEG) couplé avec une analyse EDS. Les limites de sa fiabilité et de sa reproductibilité sont présentées ici. Ceci permet de définir si la composition inclusionnaire est réellement modifiée lors de l'introduction de différents éléments d'addition et de statuer sur la pertinence de ces opérations pour modifier la microstructure de l'acier.

\section{Mode opératoire}

L'acier utilisé est une nuance G20 Mn5 (AFNOR). Il est principalement composé de 0,2\% de carbone et $1,2 \%$ de manganèse. Pour cette étude, les lingots sont réalisés dans une fonderie de laboratoire et coulés dans des moules en sable vert. La fusion a lieu dans un four à induction à moyenne fréquence d'une puissance maximale de $100 \mathrm{~kW}$ et d'une capacité de 20 litres. Le volume des pièces coulées est de 2,1 litres : suffisamment petit pour rester à l'échelle du laboratoire mais suffisamment important pour maintenir une certaine représentativité des pièces industrielles, vérifié par simulation numérique. Les dimensions du lingot sont $175 \mathrm{~mm}$ de longueur, $140 \mathrm{~mm}$ de largeur et $40 \mathrm{~mm}$ d'épaisseur. Le respect de la nuance d'acier est systématiquement vérifié à l'aide d'une analyse de spectrométrie à étincelles. 


\section{Introduction des éléments d'addition}

La première étape du processus concerne la désoxydation. La mise en place de cette opération a fourni les échantillons nécessaires pour déterminer l'efficacité de la méthode MEB-FEG couplée à une analyse EDS.

Pour le protocole opératoire de la désoxydation, deux types de désoxydation ont été réalisés lors de chaque coulée :

- Addition d'aluminium directement à l'intérieur du four pour calmer le bain selon les procédés industriels actuels avec un ratio d'un gramme d'aluminium par kilogramme d'acier

- Addition d'aluminium dans la poche de coulée (environ 0,4\%) pour agir sur la microstructure

Pour le protocole opératoire de l'inoculation, l'élément d'addition a été introduit en jet de coulée au moment du transfert de l'acier entre la poche de coulée et le moule. Trois teneurs ont été testées, une valeur nominale puis 1,5 et 2,5 fois cette valeur.

\section{Analyses au microscope électronique à balayage (MEB)}

Le microscope électronique à balayage (MEB) permet d'observer la microstructure des pièces obtenues par procédé de fonderie et d'en analyser la composition.

Les inclusions observées sont principalement les oxydes et les sulfures comme décrits précédemment. D'autres types d'inclusions existent, mais elles ne se rencontrent pas dans ce type d'acier.

\section{Méthode optique/méthode numérique}

L'analyse par microscope optique est la principale méthode utilisée en industrie. Elle consiste à analyser 20 champs de manière aléatoire à un grossissement x 500 . Les inclusions de chaque champ sont classées selon la norme NF EN 10247. La nature des inclusions est définie par différenciation des couleurs de celles-ci ; l'exactitude de l'analyse dépend donc fortement de l'opérateur.

Concernant l'analyse numérique, un logiciel intégré au MEB-FEG, AZtec, permet, par l'analyse d'image, le comptage inclusionnaire. Il fonctionne par différentiation des nuances de gris d'un échantillon pour définir la morphologie de l'inclusion et donne sa composition chimique au centre de la particule grâce à l'analyse EDS. Le grossissement choisi est $\times 500$ (identique à la méthode optique). Pour cela une préparation métallographique méticuleuse est obligatoire. Le mouvement de la caméra et les analyses EDS sont automatisés grâce au logiciel associé. Les champs scannés sont enregistrés pour une éventuelle révision ultérieure. La comparaison entre les deux méthodes est décrite dans le Tableau 1.

Tableau 1 : Comparaison de temps entre la méthode optique et la méthode MEB

\begin{tabular}{|l|l|l|l|l|l|l|}
\hline & $\begin{array}{l}\text { Surface } \\
\text { analysée }\end{array}$ & $\begin{array}{l}\text { Temps } \\
\text { d'installation }\end{array}$ & $\begin{array}{l}\text { Temps } \\
\text { d'analyse }\end{array}$ & $\begin{array}{l}\text { Type } \\
\text { d'analyse }\end{array}$ & Ratio & $\begin{array}{l}\text { Prix } \\
\text { installation }\end{array}$ \\
\hline
\end{tabular}




\begin{tabular}{|l|l|l|l|l|l|c|}
\hline MEB & $11 \mathrm{~mm}^{2}$ & $\begin{array}{l}30 \text { min (chambre sous } \\
\text { vide d'un FEG) }\end{array}$ & $4 \mathrm{~h}$ & Automatique & $\begin{array}{l}80 \\
\text { champs/h }\end{array}$ & ++ \\
\hline Optique & $0,67 \mathrm{~mm}^{2}$ & $1 \mathrm{~min}$ & $2 \mathrm{~h}$ & Manuel & $\begin{array}{l}10 \\
\text { champs/h }\end{array}$ & - \\
\hline
\end{tabular}

Comme le système est automatisé, le gain de temps est important par rapport à une observation conventionnelle sous un microscope optique. La méthode optique est basée sur le comptage d'inclusions sur 20 champs aléatoires de même taille que sur la Figure 4. La zone couverte est alors moins large qu'avec le logiciel et la précision de détection des inclusions est subjective pour l'observateur. Pour 20 champs analysés en méthode optique, 80 seront analysés en méthode MEB. De plus la méthode MEB fournit la composition chimique de chaque particule. Cela réduit le risque de confondre une porosité avec une inclusion.

\section{Procédure d'analyse détaillée sous MEB-FEG}

\section{Première étape}

La première étape de cette analyse est l'acquisition du premier champ (Figure 4). La taille du champ correspond à la taille de la méthode optique. Les niveaux de gris sont définis manuellement de manière à détecter un maximum de particules quel que soit leur type. Le champ étudié, tel que défini sur la Figure 4, doit être représentatif de l'échantillon total afin de détecter tous les types d'inclusions. Cette première étape est donc cruciale pour la réussite d'un comptage inclusionnaire automatisé.

Un seuil minimal de détection a été utilisé afin de limiter le temps d'analyse et la quantité de données. On considère que les particules avec un diamètre équivalent inférieur à $2 \mu \mathrm{m}$ n'auront pas d'impact sur l'acier que ce soit au niveau de la microstructure ou des propriétés mécaniques[8].

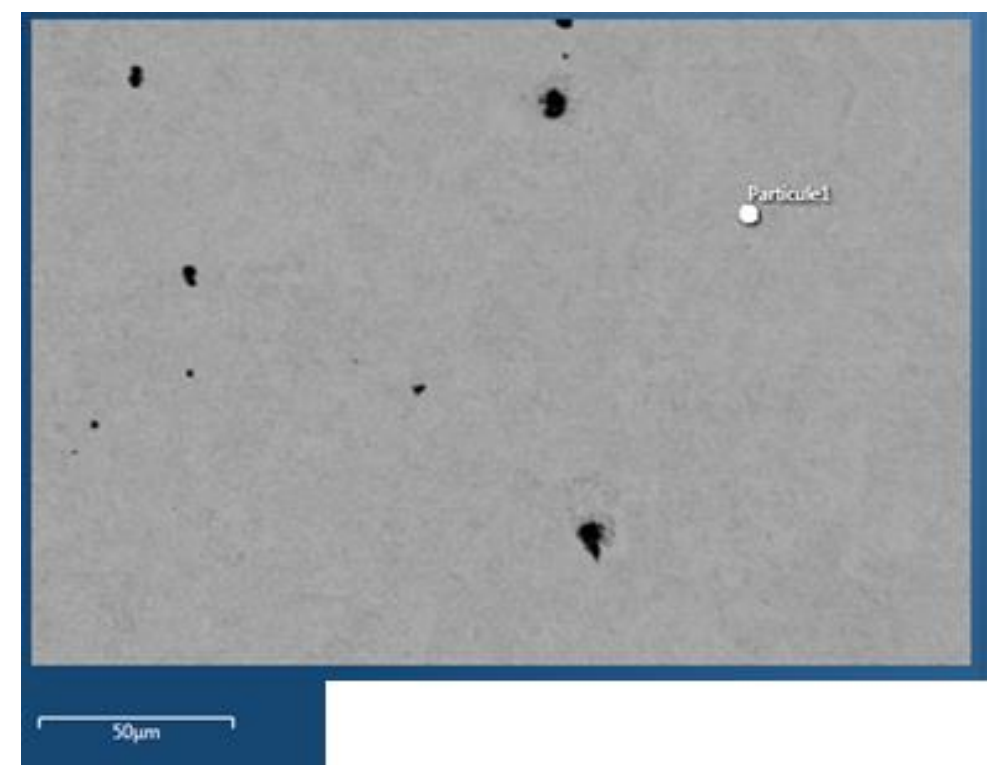

Figure 4 : Exemple de champ élémentaire. Grossissement x500 
La deuxième étape est l'automatisation. Une surface d'analyse est définie par l'opérateur, ici $11 \mathrm{~mm}^{2}$. Le logiciel divise cette surface en champs élémentaires (même champ élémentaire que pour les méthodes optiques) puis détecte et analyse chaque particule à l'intérieur de chaque champ élémentaire. Plusieurs surfaces peuvent être examinées comme le montre la Figure 5. Cela signifie que différents échantillons peuvent être analysés pendant l'automatisation. Sur la Figure 5 se trouve une surface dont l'analyse est finie et une surface dont l'analyse est en cours. Les tailles des zones et surfaces ont été définies précédemment.

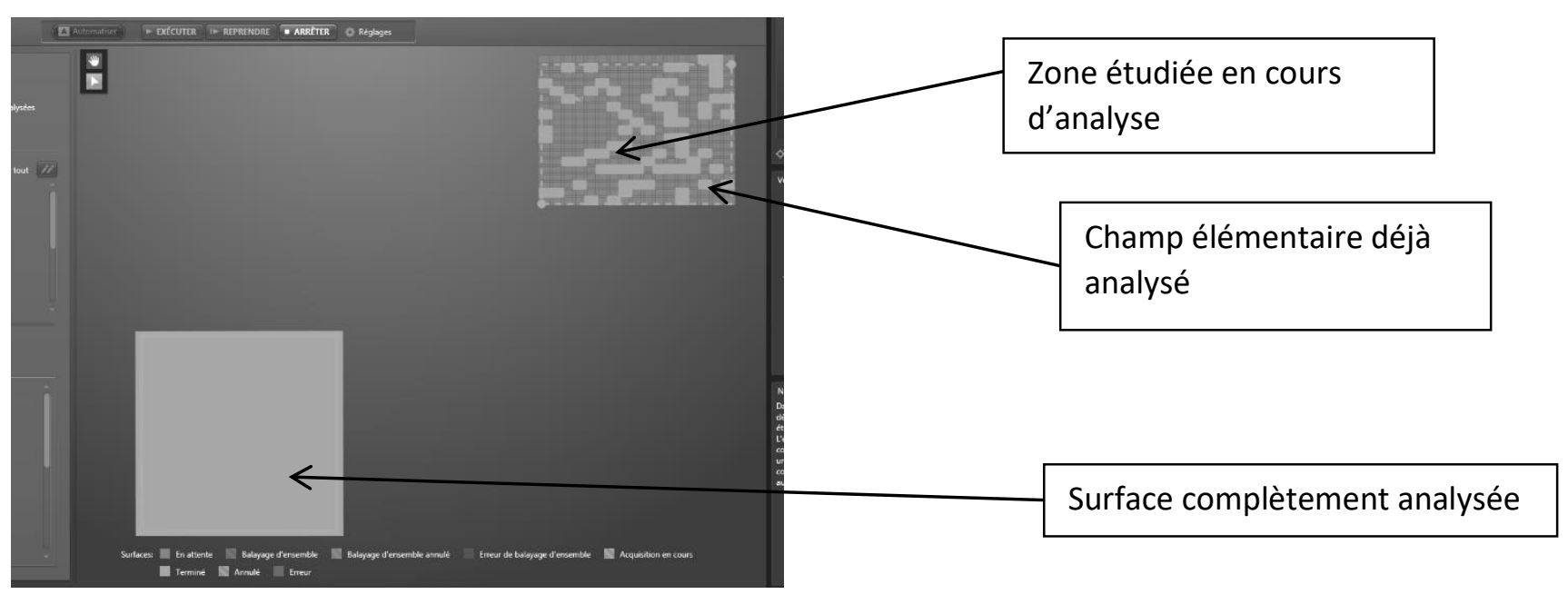

Figure 5 : Surface étudiée aux différentes étapes d'analyse

\section{Troisième étape}

La dernière étape est la classification des particules grâce à des analyses chimiques. La méthode de classification des particules est réalisée en fonction de leur composition chimique et de leur morphologie dans le cas des sulfures. La classification de la morphologie est faite grâce à la détermination d'un seuil du rapport des diamètres équivalents. Elle est comparable à la classification utilisée dans les logiciels concurrents [10]. Cette classification s'appliquera à toutes les particules après analyse et est la même pour chaque surface évaluée. Le logiciel identifie également la forme des inclusions notamment pour les sulfures et les classifie en fonction de leur type. Les types ont été décrits précédemment.

Si la composition en fer est supérieure à $70 \%$, on considère qu'il s'agit de porosités.

Ensuite les différents types d'inclusion sont définis en fonction de leur composition chimique et de leur facteur de forme (rapport grande diagonale sur petite diagonale). Le facteur de forme va permettre de différencier les sulfures de type II des autres types de sulfures. Le logiciel ne permet pas de distinguer les sulfures de type I des sulfures de type III.

La classification se fait manuellement et comme suit :

- Sulfure (Type I et III) : teneur en soufre supérieur à $15 \%$ et facteur de forme inférieur à 1,5 (tendance à être ronds)

- Sulfure Type II : teneur en soufre supérieur à $15 \%$ et facteur de forme supérieur à 1,5 
- Oxyde d'aluminium : Teneur en aluminium supérieur à 15\%, ce sont les types d'oxydes les plus courants de cet acier

- Oxydes de silicium : teneur en silicium supérieure à 15\%. Critère appliqué après tous les autres critères.

\section{Etude de l'influence du système de mesure sur le comptage inclusionnaire}

Une surface type d'un échantillon seulement désoxydé (ajout d'aluminium) est analysée. La surface est de $11 \mathrm{~mm}^{2}$. L'échantillon est préparé comme suit : mise en résine carbone et polissage. La surface est repérée avec une pointe diamant. L'échantillon est ensuite introduit dans le MEB-FEG. La surface est analysée 10 fois puis l'échantillon est retiré du MEB. Cette opération est répétée 3 fois pour un total de 30 analyses de la surface. Une étude statistique est réalisée sur les résultats en fonction du nombre et du type d'inclusions. La distribution des particules doit être normale pour pouvoir appliquer les lois statistiques [11]. L'exemple des Figure 6 et Figure 7 montre effectivement une répartition normale des données selon le test de Shapiro Wilk[12] ce qui simplifie grandement les prochains calculs.

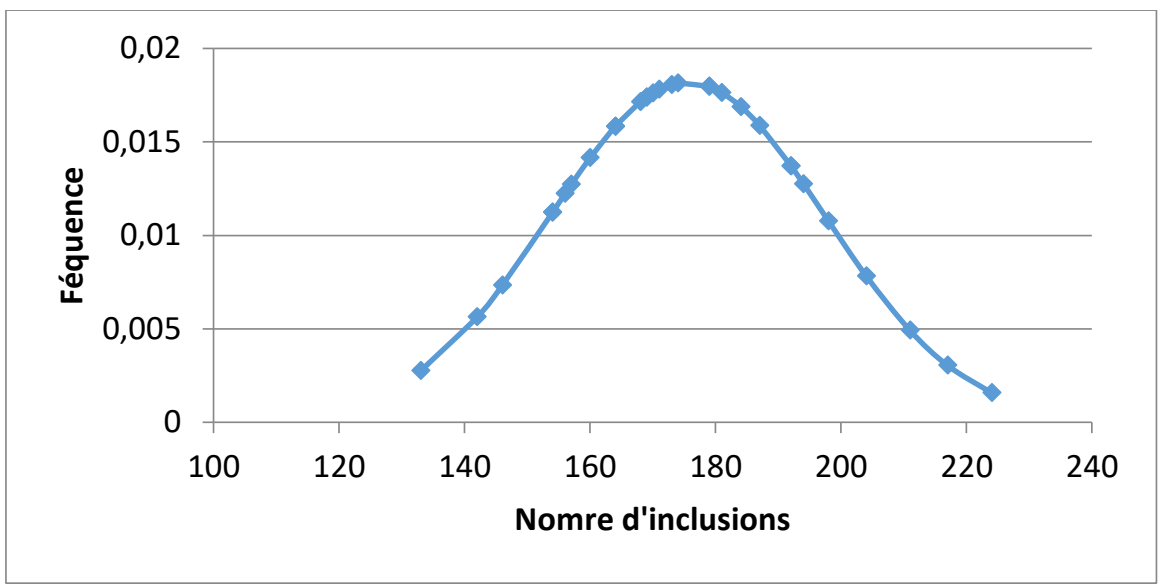

Figure 6 : Distribution des oxydes 


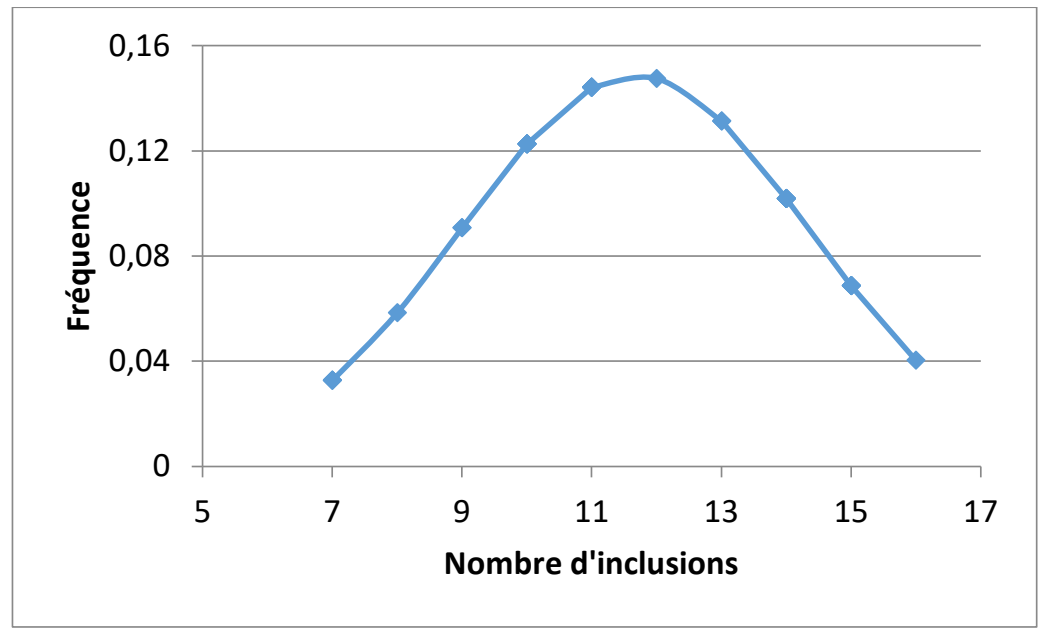

\section{Figure 7 : Distribution du total des sulfures}

Un calcul d'écart type est appliqué aux données. L'écart type sert à mesurer la dispersion desdites données et ainsi obtenir un intervalle de tolérance pour chaque type d'inclusion. Afin d'obtenir une précision suffisante il a été décidé de choisir cet intervalle de tolérance de $4 \sigma$ permettant d'englober 95\% des données mesurées. Le Tableau 2 récapitule la façon d'obtenir ce dernier.

Tableau 2 : résultats de l'étude statistique du nombre et du type de particules analysées

\begin{tabular}{|l|l|l|l|l|l|}
\hline & Oxydes & $\begin{array}{l}\text { Sulfures type } \\
\text { II }\end{array}$ & $\begin{array}{l}\text { Sulfures type I et } \\
\text { III }\end{array}$ & Sulfures totaux & $\begin{array}{l}\text { Total des } \\
\text { particules }\end{array}$ \\
\hline moyenne & 11,7 & 20,5 & 155 & 175 & 809 \\
\hline $\begin{array}{l}\boldsymbol{\sigma}=\text { écart } \\
\text { type exp }\end{array}$ & 2,7 & 4,2 & 17,9 & 20,8 & 36,7 \\
\hline $\mathbf{4 \sigma}$ & 10,7 & 16,6 & 71,5 & 83,3 & 146,9 \\
\hline & $\mathrm{m}=11,7 \pm 5,3$ & $\mathrm{~m}=20,5 \pm 8,3$ & $\mathrm{~m}=155 \pm 35$ & $\mathrm{~m}=175 \pm 41$ & $\mathrm{~m}=809 \pm 73$ \\
\hline
\end{tabular}

La Figure 8 extrapole les intervalles de tolérances à partir du Tableau 2 et pour tous les nombres de particules. Cela crée une zone à éviter s'il l'on veut observer des différences significatives entre 2 inoculants différents. La zone déterminée entre les deux courbes de la Figure 8 est la zone à éviter pour obtenir des résultats significativement différents entre deux résultats. 


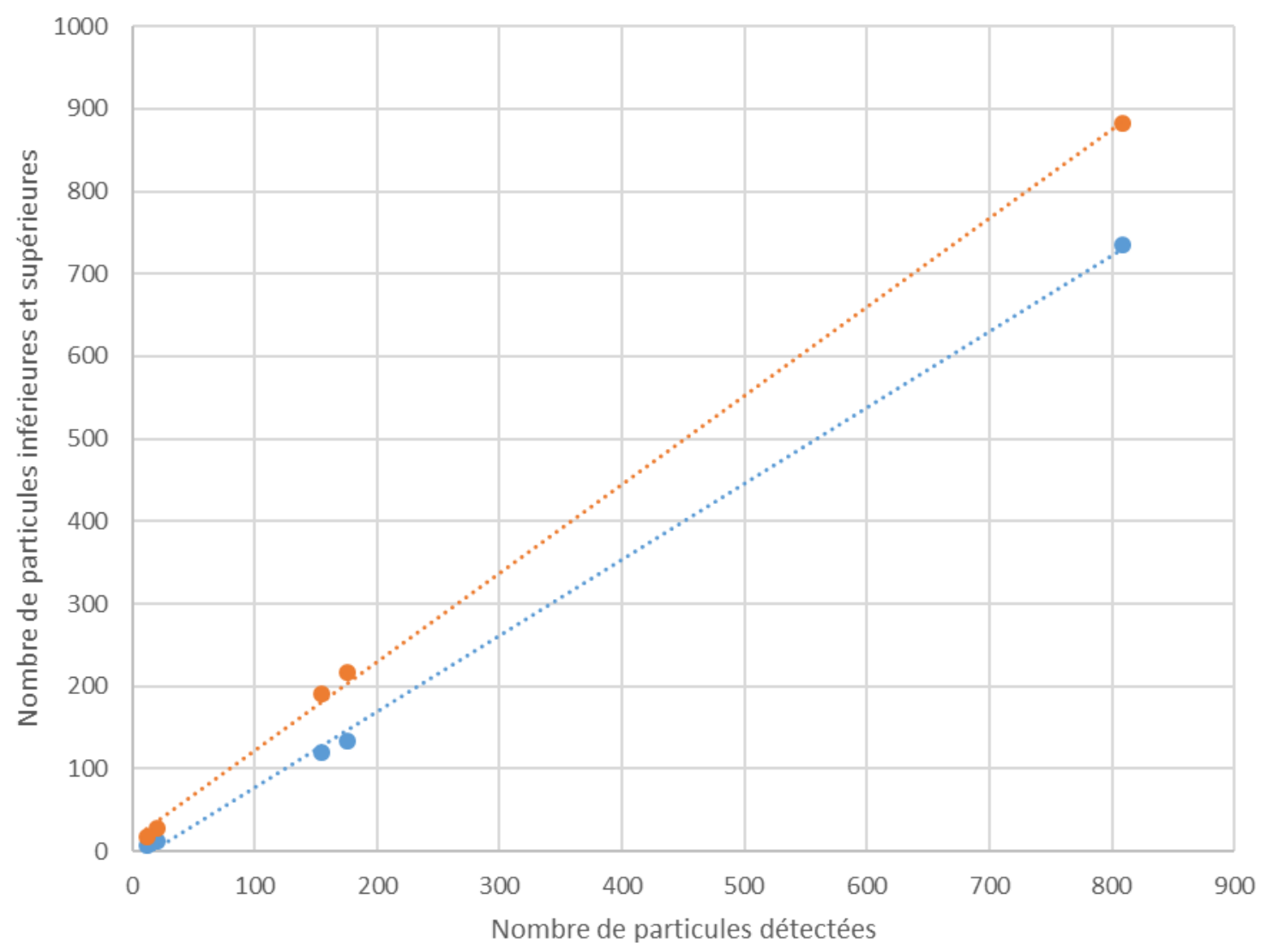

Figure 8 : Intervalles de tolérance en fonction du nombre de particules

La Figure 9 montre que plus le nombre de particules détectées est grand, plus l'écart type sera faible par rapport à la moyenne des particules détectées. La précision du nombre de particules détectées est donc plus importante.

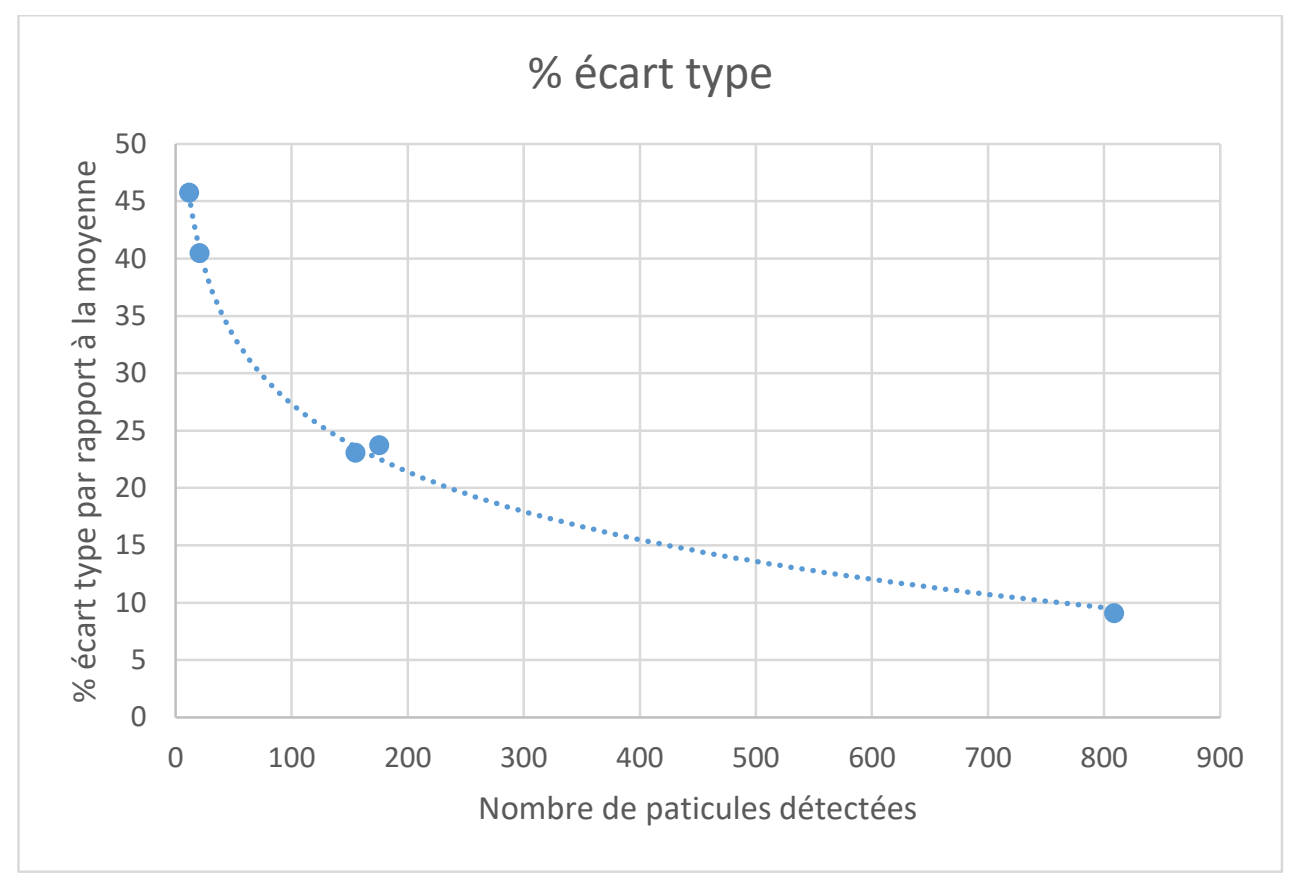

Figure 9 : Evolution de l'écart type en fonction du nombre de particules 
Les analyses statistiques étant faites, la comparaison entre les différents ensembles inclusionnaires est maintenant possible. Grâce aux intervalles de tolérance déterminés précédemment il est possible d'observer s'il existe des différences significatives entre les différents éléments d'addition introduits dans l'acier.

\section{Effets des inoculants sur la propreté inclusionnaire}

Grâce aux études préliminaires, il est possible de comparer les effets des différents éléments ajoutés sur l'ensemble inclusionnaire de l'acier. La Figure 10 répertorie les résultats concernant le nombre de sulfures en fonction de la teneur en soufre résiduel pour chaque inoculant.

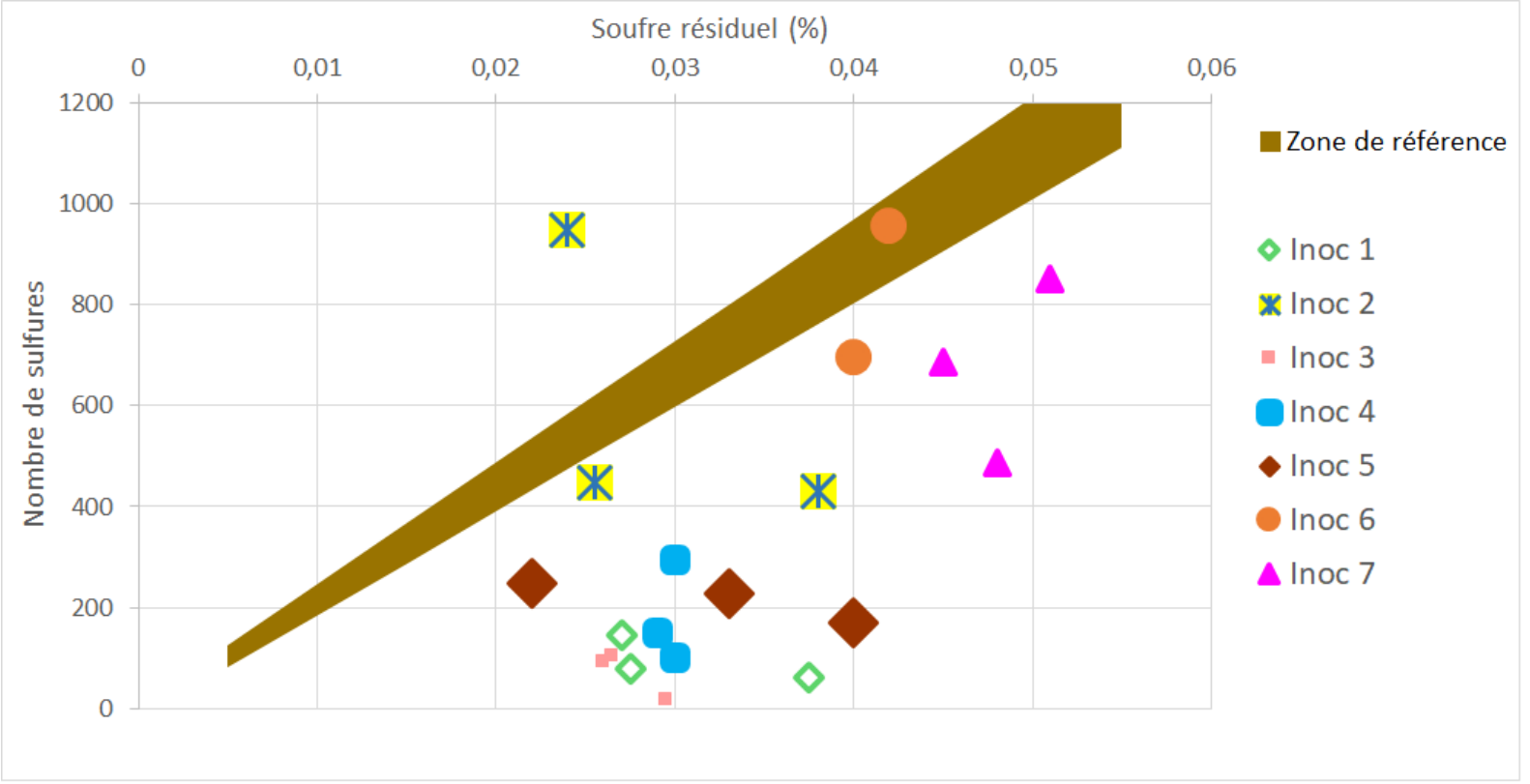

Figure 10 : Quantité d'inclusions de sulfure en fonction du soufre résiduel pour chaque élément ajouté (Aluminium ou inoculant) 


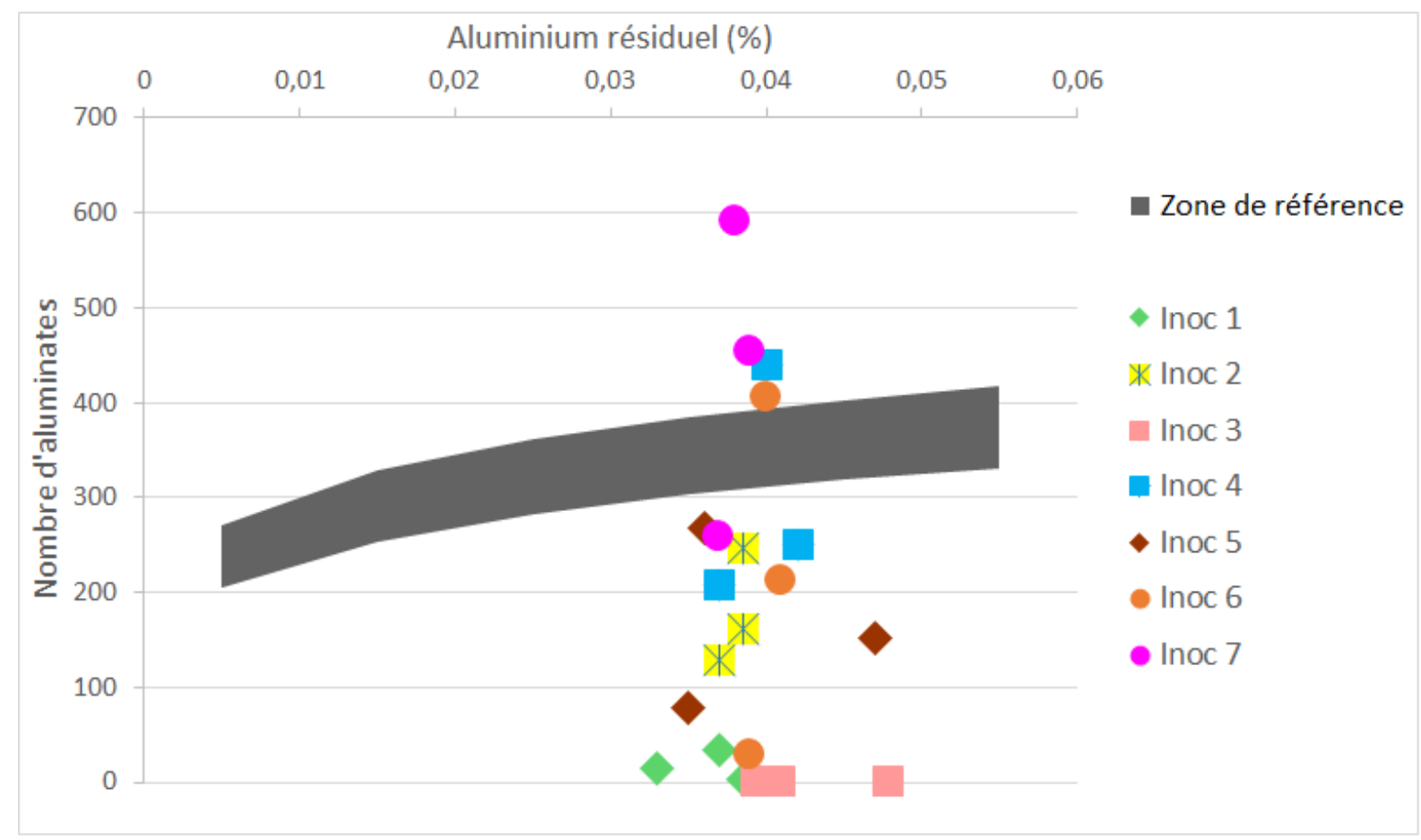

Figure 11 : Quantité d'inclusions d'oxyde en fonction de l'aluminium résiduel pour chaque élément ajouté (Aluminium ou inoculant)

La zone de référence sur la Figure 10 est déterminée par les essais concernant uniquement la désoxydation donc avec un ajout d'aluminium seul. La plupart des inoculants sont en dehors de la zone référence. Ce sont donc des résultats significativement différents par rapport aux essais d'aluminium seul.

L'intérêt d'ajouter d'autres composants est de réduire la quantité de sulfures dans l'acier. Cet effet de réduction est observé pour la plupart des inoculants même en prenant en compte les intervalles de tolérances définis plus haut. De part ces résultats, il est possible de conclure deux points : le système d'analyse est suffisamment performant pour comparer les différents échantillons et la méthode d'inoculation fait chuter le nombre de sulfures. Cette tendance est confirmée dans le cas des oxydes par la Figure 11 où la ligne de référence est cette fois-ci une fonction logarithmique mais qui est toujours défini par les essais de désoxydation sans ajout d'élément inoculant. Tous les résultats sont en dehors de cette zone. Ils sont significativement différents des essais de référence. Les résultats en dessous de la zone montrent une amélioration de la propreté inclusionnaire concernant les oxydes.

\section{Conclusion}

D'abord cette étude présente l'utilisation et les limites de la méthode d'analyse par MEB-FEG couplé à un logiciel d'automatisation du processus. La méthode numérique de comptage d'inclusion permet de couvrir une surface plus importante en moins de temps que la méthode optique traditionnelle. Elle permet également d'obtenir toutes les compositions chimiques de toutes les particules. Cette méthode réduit également le biais de l'observateur lors du comptage d'inclusion. Cela évite de manquer des inclusions ou de compter des porosités en tant qu'inclusions. Grâce à l'étude statistique il a été permis de définir les intervalles de tolérance pour la détection de particules dans l'acier. Ainsi la pertinence des résultats ultérieurs est appréciée. Les résultats de l'étude statistique sont réutilisables pour d'autres études impliquant la détection de particules, par exemple avec d'autres aciers que celui utilisé. 
Ensuite l'étude montre la comparaison entre différents éléments ajoutés concernant les inclusions notamment les sulfures dans un acier faiblement allié. En fonction de l'inoculant ajouté on observe une diminution plus ou moins importante de la quantité de sulfures. Couplé aux intervalles de tolérances définis par l'étude statistique il est déterminé que ces diminutions sont significatives. Il y a donc une véritable amélioration possible de l'acier grâce à la méthode d'inoculation. D'autres essais complémentaires seront réalisés afin de confirmer cette tendance. Les résultats les plus pertinents pourront ensuite être testés sur des coulées de taille industrielle.

\section{Remerciements}

Cette étude est financée par SAFE Metal

\section{Références}

[1] G. Zhang and K. Chou, "Deoxidation of Molten Steel by Aluminum," J. Iron Steel Res. Int., vol. 22, no. 10, pp. 905-908, 2015.

[2] Y. Li et al., "Effect of Zr-Ti combined deoxidation on the microstructure and mechanical properties of high-strength low-alloy steels," Mater. Sci. Eng. A, vol. 659, pp. 179-187, 2016.

[3] V. A. Golubtsov, L. G. Shub, A. A. Deryabin, and R. G. Usmanov, "Treating steel outside the furnace more efficiently," Metallurgist, vol. 50, no. 11-12, pp. 634-637, 2006.

[4] A. Rist, M.-F. Ancey-Moret, C. Gatellier, and P.-V. Riboud, Équilibres Thermodynamiques En Sidérurgie, vol. 33, no. 0. 1974.

[5] Z. LI, C. LIU, Q. SUN, and M. JIANG, "Effect of Deoxidation Process on Distribution Characteristics of Inclusions in Silicon Steel Slabs," J. Iron Steel Res. Int., vol. 22, no. Supplement 1, pp. 104-110, 2015.

[6] J. C. Yarwood, M. C. Flemings, and J. F. Elliott, "Inclusion formation in the Fe-O-S system," Metall. Trans., vol. 2, no. 9, pp. 2573-2582, 1971.

[7] L. Zhang and G. St, "State of the Art in the Control of Inclusions during Steel Ingot Casting," Mater. Metall. Trans., vol. 37, 2006.

[8] ETIF, "Méthode micrographique de détermination de la teneur en inclusions non métalliques des aciers moulés," NF EN 10247, 2007.

[9] A. Pokorny and J. Pokorny, "Inclusions non métalliques dans l'acier," Tech. l'Ingenieur, vol. 33, no. M220, pp. 1-43, 1998.

[10] E. Hénault, "Method of Automatic Characterization of Inclusion Population by a SEM-FEG / EDS / Image," JEOL News, vol. 41, no. 1, pp. 1-3, 2006.

[11] O. Castéra, "La loi normale," Univ. paris ouest, pp. 1-43, 2012.

[12] S. S. Shapiro and M. B. Wilk, "An analysis of variance test for normality (complete samples)," Boimetrika, vol. 52, no. 3/4, pp. 591-611, 1965. 